\title{
Fractional integrals of modular forms
}

\author{
by \\ Wladimir de Azevedo Pribitkin (Staten Island, NY)
}

1. Introduction. Among the wealth of lovely dualities inherent in the theory of automorphic forms, perhaps the most captivating is the one connecting forms of weight $k$ and $2-k$. Sometimes called Serre Duality, at its core this link reveals simply that the $(1-k)$ th derivative of an automorphic form of integer weight $k<1$ is itself an automorphic form of weight $2-k$. This implies, of course, that the $(k-1)$-fold integral of an automorphic form of integer weight $k>1$ is also an interesting automorphic object. This function, called an Eichler integral, transforms like a form of weight $2-k$ modulo a period polynomial of degree at most $k-2$ in its modular relation. The validity of these statements, which hold for integer weights only, will be explained below. But what about other real weights?

In 1993 it occurred to the author that fractional integration could be a key tool for mapping forms of noninteger weight to certain integrals of dual weight. Some of the results presented here date back to 1994, whereas others are more recent. For simplicity we work on the full modular group. All of the theorems extend to more general groups, but there certain entire forms (such as Eisenstein series) of noninteger weight also have to be accomodated.

In this paper we first consider the effect of fractional integration (the appropriate number of times, perhaps negative) on a modular form of arbitrary weight and multiplier system, with a possible principal part at infinity. We call the resulting function a "generalized Eichler integral", and in Section 2 we describe fully its behavior under modular transformations. Although rather complicated, this transformation law simplifies dramatically for generalized Eichler integrals of cusp forms (see Corollary 3). And in Section 3 we show that this relation also reduces somewhat when one starts with Poincaré series having a pole at $\infty$.

In Section 4 we demonstrate a rather simple nexus between these "polar Poincaré series" and Niebur modular integrals (the definition of which is recalled in the same section). This mapping from polar Poincaré series to

2000 Mathematics Subject Classification: Primary 11F11. 
Niebur modular integrals consists of combining fractional integration with conjugation of the multiplier system. It is almost a triviality to show that the resulting function, which we call a "conjugate Eichler integral", is in fact a Niebur modular integral. Connected with this type of integral there is yet another function, an "auxiliary integral", which we examine in Section 5. We conclude the paper with some natural questions which are not addressed here. We also mention that recently Zagier $[17,18]$ has studied the semi-integrals (i.e., fractional integrals of half-integer order) of certain entire modular forms of half-integer weight. His work, which is related to some of our previous results on Niebur modular integrals of small weight [9-11], includes beautiful new identities that are linked to enumeration of invariants arising out of knot theory. We feel that there remains much to be uncovered along the lines of our investigation.

We now provide the promised substantiation of the rudimentary facts concerning the well known case of integer weight forms. Let $F$ be any function of the complex variable $z$ and assume that it can be differentiated $r$ times, where $r$ is a nonnegative integer. Then it is not difficult to verify (by induction on $r$ ) that

$$
\begin{aligned}
& F^{(r)}(V z) \\
& \quad=\sum_{j=0}^{r}\left(\begin{array}{l}
r \\
j
\end{array}\right) \frac{\Gamma(w+r)}{\Gamma(w+j)} \gamma^{r-j}(\gamma z+\delta)^{w+r+j} \frac{d^{j}}{d z^{j}}\left\{(\gamma z+\delta)^{-w} F(V z)\right\},
\end{aligned}
$$

where $V=\left(\begin{array}{ll}* & * \\ \gamma & \delta\end{array}\right) \in \operatorname{SL}(2, \mathbb{C})$ and $w$ is a complex number. Setting $w=1-r$ we obtain the important special case

$$
(\gamma z+\delta)^{-r-1} F^{(r)}(V z)=\frac{d^{r}}{d z^{r}}\left\{(\gamma z+\delta)^{r-1} F(V z)\right\}
$$

which was observed by Bol [1] in 1949. The latter equality can also be proved by invoking Cauchy's Integral Formula. Obviously, if $F$ is an automorphic form of integer weight $k<1$ on a suitable group $\Gamma$, then Bol's Identity tells us that

$$
F^{(1-k)}(V z)=(\gamma z+\delta)^{2-k} F^{(1-k)}(z), \quad \forall V \in \Gamma,
$$

and secures the assertions in our opening paragraph. If needed a multiplier system in weight $k$ may be inserted gratis into the above equality. Since $k$ and $2-k$ share the same parity, the claims still hold. (And this is actually a crucial point in the context of fractional differentiation and integration of noninteger weight forms.)

We add that in 1953 Maass [5] introduced his weight-raising operator

$$
\delta_{w}=\frac{\partial}{\partial z}+\frac{w}{2 y i},
$$

which maps a form of weight $w$ to a form of weight $w+2$. (To some readers 
this may be more familiar then Bol's work.) Here $y=\operatorname{Im} z, w$ is complex and the form need not be holomorphic. Now, if we consider $\delta_{w}^{r}=\delta_{w+2 r-2} \circ$ $\cdots \circ \delta_{w+2} \circ \delta_{w}$ for $r \in \mathbb{Z}^{+}$, and $\delta_{w}^{0}$ equal to the identity, it is straightforward to check that

$$
\delta_{w}^{r}=\sum_{j=0}^{r}\left(\begin{array}{l}
r \\
j
\end{array}\right) \frac{\Gamma(w+r)}{\Gamma(w+j)}(2 y i)^{j-r} \frac{\partial^{j}}{\partial z^{j}}
$$

and that it maps a form of weight $w$ to one of weight $w+2 r$. So, we see anew that $\delta_{k}^{1-k}=d^{1-k} / d z^{1-k}$ maps a form to a form. For much more on classical Eichler integrals, we suggest the seminal works of Eichler [2] and Shimura [15], as well as the paper by Kohnen \& Zagier [4].

2. Generalized Eichler integrals. We let

$$
f(\tau)=\sum_{n=-\mu}^{\infty} a_{n} e^{2 \pi i(n+\kappa) \tau}, \quad \tau \in \mathcal{H} \text { (upper half-plane), }
$$

be a modular form of real weight $k$ and multiplier system (MS) $v$ on $\Gamma(1)$, the full modular group. The function $v$ maps matrices to the unit circle and the parameter $\kappa$ is determined by $v(S)=e^{2 \pi i \kappa}, 0 \leq \kappa<1$, where $S=\left(\begin{array}{ll}1 & 1 \\ 0 & 1\end{array}\right)$. Recall that the Fourier expansion (2) is a consequence of the definition of a modular form, which requires $f$ to be holomorphic in $\mathcal{H}$, meromorphic at $\infty$ and to satisfy the modular relation

$$
f(\tau)-\bar{v}(V)(\gamma \tau+\delta)^{-k} f(V \tau)=0
$$

for all $V=\left(\begin{array}{cc}* & * \\ \gamma & \delta\end{array}\right) \in \Gamma(1)$ and $\tau \in \mathcal{H}$. Throughout our work we employ the convention $-\pi \leq \arg (\cdot)<\pi$ to dictate branch selection. We denote the principal part at $\infty$ of $f$ by $f_{\infty}$. If $f$ has no constant term, then we say that it is "constant-free". Note that on the full modular group $f$ is constant-free except perhaps when $k$ is an even integer and $v \equiv 1$. (We remark, however, that a form on $\Gamma(1)$ of weight 2 is always constant-free. To see this, recall that nontrivial entire forms on $\Gamma(1)$ of weight 2 and $v \equiv 1$ do not exist, but constant-free forms of this weight and MS, with any prescribed principal part, do exist on $\Gamma(1)$.) Below we shall study the effect of a certain operator on $f$.

Specifically, we consider the $(k-1)$ th fractional integral of $f$, which we define by

$$
F(\tau)=\sum_{n=-\mu}^{\infty} \frac{a_{n}}{[-2 \pi i(n+\kappa)]^{k-1}} e^{2 \pi i(n+\kappa) \tau}, \quad \tau \in \mathcal{H},
$$

where $f$ is assumed to be constant-free for $k>1$. (This is also the $(1-k)$ th fractional derivative of $f$.) We call $F$ the generalized Eichler integral of $f$. When $k$ is an integer greater than 1 , then $F+$ possibly a constant term 
becomes the familiar Eichler integral of $f$. This term, which we omit for succinctness, is discussed briefly at the end of the next two sections. Likewise, when $k$ is a nonpositive integer, then $F$ reduces to the "Bol derivative" of $f$. (Actually, these statements concerning integer weights are true modulo a possible constant factor.) Observe that we have not chosen a normalization and, moreover, that our definition is in general not equivalent (in the sense of merely multiplying by a constant) to one which replaces the denominator $[-2 \pi i(n+\kappa)]^{k-1}$ with $[2 \pi i(n+\kappa)]^{k-1}$. For cusp forms the two definitions are equivalent, however. It is clear that $F$ is holomorphic in $\mathcal{H}$ and meromorphic at $\infty$. Its modular behavior, which is far from obvious, requires that we possess an integral representation for $F$.

Our formulation essentially coincides with Weyl's notion [16] of fractional integration. (For more on the fractional calculus, we recommend [6], although everything we use here is rather self-evident.) Specifically, for $1<k<2$, it is true that

$$
F(\tau)=\frac{1}{\Gamma(k-1)} \int_{\tau}^{\infty} f(z)(z-\tau)^{k-2} d z,
$$

where the path of integration is horizontal. For other values of $k$ convergence difficulties arise and it is wise to separate $f$ into its analytic and principal parts. The former can be integrated along any path which goes up to infinity in $\operatorname{Im} z>\operatorname{Im} \tau$, and the latter along any path which goes down to infinity in $\operatorname{Im} z<\operatorname{Im} \tau$. (That is, for $k>1$, we simply bend the path in (5) up or down, respectively.) The precise integral representations which we use to establish the following two theorems are given by (7) for $k>1$ and by (11) for $k \leq 1$.

THEOREM 1. Let $f$ be a constant-free modular form of real weight $k>1$, and denote by $F$ its generalized Eichler integral, given by the Fourier expansion (4). Also, let $f_{\infty}$ be the (possibly trivial) principal part of $f$. Then $F$ satisfies the relation

$$
\begin{aligned}
& F(\tau)-\bar{v}(V)(\gamma \tau+\delta)^{k-2} e^{-2 \pi i N_{1} k} F(V \tau) \\
& =\frac{1}{\Gamma(k-1)}\left\{\int_{V^{-1}(\infty)}^{i \infty}\left[f(z)-f_{\infty}(z)-\frac{f_{\infty}(V z)}{v(V)(\gamma z+\delta)^{k}}\right](z-\tau)^{k-2} e^{2 \pi i N_{2} k} d z\right. \\
& \quad+e^{-2 \pi i N_{1} k} \int_{V^{-1}(\infty)}^{-i \infty}\left[e^{2 \pi i k} f_{\infty}(z)+\frac{f_{\infty}(V z)}{v(V)(\gamma z+\delta)^{k}}\right](z-\tau)^{k-2} d z \\
& \quad+\left(1-e^{-2 \pi i N_{1} k}\right) \int_{\tau}^{i \infty} \frac{f_{\infty}(V z)}{v(V)(\gamma z+\delta)^{k}}(z-\tau)^{k-2} d z \\
& \left.\quad+\left(1-e^{2 \pi i\left(1-N_{1}\right) k}\right) \int_{\tau}^{-i \infty} f_{\infty}(z)(z-\tau)^{k-2} d z\right\}
\end{aligned}
$$


for all $V=\left(\begin{array}{ll}* & * \\ \gamma & \delta\end{array}\right) \in \Gamma(1), \gamma>0$, and $\tau \in \mathcal{H}$, where

$$
\begin{aligned}
& N_{1}=N_{1}(\tau, V)= \begin{cases}1 & \text { if } \operatorname{Re} \tau \leq V^{-1}(\infty), \\
0 & \text { if } \operatorname{Re} \tau>V^{-1}(\infty),\end{cases} \\
& N_{2}=N_{2}(z-\tau)= \begin{cases}1 & \text { if }-\pi \leq \arg (z-\tau)<-\pi / 2, \\
0 & \text { if }-\pi / 2 \leq \arg (z-\tau)<\pi .\end{cases}
\end{aligned}
$$

(All the paths of integration are vertical.)

Before we prove Theorem 1, we make some remarks. We first observe that the function on the left side of the above relation (sort of a period for $f$ ) is holomorphic in $\mathcal{H} \backslash\left(V^{-1}(\infty), i \infty\right)$. We also comment on the case $\gamma \leq 0$, which is not included above, but can be easily reckoned. If $\gamma=0$ (i.e., $V$ is a translation), then clearly $F(V \tau)=v(V) F(\tau)$. Also, since $V$ and $-V$ induce the same transformation, the relations for $\gamma<0$ and $\gamma>0$ are equivalent to each other. To actually write down one from the other, recall that the consistency condition for multiplier systems tells us that $v(V)(\gamma \tau+\delta)^{k}=v(-V)(-\gamma \tau-\delta)^{k}$. For $\gamma \neq 0$ this implies that $\bar{v}(V) v(-V)=$ $(-\gamma \tau-\delta)^{-k}(\gamma \tau+\delta)^{k}=e^{\operatorname{sign}(\gamma) \pi i k}$ (since the argument of -1 is $-\pi$ ) and hence

$$
\bar{v}(V)(\gamma \tau+\delta)^{k-2}=e^{\operatorname{sign}(\gamma) 2 \pi i k} \bar{v}(-V)(-\gamma \tau-\delta)^{k-2} .
$$

The rest is obvious.

Proof of Theorem 1. A calculation shows that for $k>1$,

$$
\begin{aligned}
F(\tau)= & \frac{1}{\Gamma(k-1)}\left\{\int_{\tau}^{i \infty}\left[f(z)-f_{\infty}(z)\right](z-\tau)^{k-2} d z\right. \\
& \left.+\int_{\tau}^{-i \infty} f_{\infty}(z)(z-\tau)^{k-2} d z\right\} .
\end{aligned}
$$

The term by term integration is routinely justified by the favorable convergence properties of the Fourier expansion of $f$. This integral representation is our starting point for establishing the transformation behavior of $F$.

Although the relation governing this behavior is complicated, its proof is largely an exercise in branching considerations and contour deformations. From (7) it follows that

$$
\begin{aligned}
F(V \tau)= & \frac{1}{\Gamma(k-1)}\left\{\int_{\tau}^{V^{-1}(\infty)}\left[f(V u)-f_{\infty}(V u)\right](V u-V \tau)^{k-2} \frac{d u}{(\gamma u+\delta)^{2}}\right. \\
& \left.+\int_{\tau}^{V^{-1}(\infty)} f_{\infty}(V u)(V u-V \tau)^{k-2} \frac{d u}{(\gamma u+\delta)^{2}}\right\}
\end{aligned}
$$


where the two paths of integration form the unique circle (or line) orthogonal to the real axis and passing through $\tau$ and $V^{-1}(\infty)$. The first contour is a hyperbolic geodesic (euclidean minor arc or line segment) in $\mathcal{H}$, whereas the second is its complement (euclidean major arc or union of two rays) which originates in $\mathcal{H}$ but enters $V^{-1}(\infty)$ through the lower half-plane. Observe that along the first contour $\arg (V u-V \tau)$ equals $\pi / 2$, but along the second it equals $-\pi / 2$. Now,

$$
(V u-V \tau)^{k-2}=\frac{(u-\tau)^{k-2}}{(\gamma u+\delta)^{k-2}(\gamma \tau+\delta)^{k-2}} e^{2 \pi i N k},
$$

where

$$
2 \pi N=\arg (V u-V \tau)-\arg (u-\tau)+\arg (\gamma u+\delta)+\arg (\gamma \tau+\delta)
$$

and $N$ is an integer-valued function to be determined shortly. Using (3) and (9) in (8) we obtain

$$
\begin{aligned}
& -\bar{v}(V)(\gamma \tau+\delta)^{k-2} F(V \tau) \\
& =\frac{1}{\Gamma(k-1)}\left\{\int_{V^{-1}(\infty)}^{\tau}\left[f(u)-\frac{f_{\infty}(V u)}{v(V)(\gamma u+\delta)^{k}}\right](u-\tau)^{k-2} e^{2 \pi i N k} d u\right. \\
& \left.\quad+\int_{V^{-1}(\infty)}^{\tau} \frac{f_{\infty}(V u)}{v(V)(\gamma u+\delta)^{k}}(u-\tau)^{k-2} e^{2 \pi i N k} d u\right\},
\end{aligned}
$$

where the paths are unaltered except for their reversed orientations.

We next find the value of $N$ on the two contours. For arbitrary $\gamma$, $|2 \pi N|<4 \pi$ and so $N \in\{-1,0,1\}$. It is best to consider the two paths together. Recall that their union is a circle (or line). We start with $u$ in the lower half-plane, where clearly $N=0$. Assume from now on that $\gamma>0$. As we move up through the branch point at $u=V^{-1}(\infty)$, we see that $\arg (V u-V \tau)+\arg (\gamma u+\delta)$ jumps from $-\pi / 2-\pi / 2$ to $\pi / 2+\pi / 2$ and hence $N$ jumps from 0 to 1 . Similarly, if the branch cut emanating from $u=V^{-1}(\infty)$ intersects the circle, then as we move up through this antipodal point, we see that $\arg (\gamma u+\delta)$ changes from $-\pi$ to $\pi$ and so $N$ increases from 0 to 1 . Additionally, if the branch cut from $u=\tau$ intersects the circle, then as we move clockwise through this point of intersection, we find that $\arg (u-\tau)$ leaps from $-\pi$ to $\pi$ and so $N$ decreases from 1 to 0 . No other jump discontinuities are possible, and so this completely specifies $N$. We organize these facts, which are valid for $\gamma>0$, as follows. For the first contour, $N=1$ unless $\operatorname{Re} \tau>V^{-1}(\infty)$ and $\operatorname{Im} u>\operatorname{Im} \tau$, in which case $N=0$. Analogously, for the second contour, $N=0$ unless $\operatorname{Re} \tau<V^{-1}(\infty)$ and $0<\operatorname{Im} u \leq \operatorname{Im} \tau$, in which case $N=1$. (One could perform a parallel analysis for $\gamma<0$, in which case the value $N=-1$ would arise.) 
The above facts and Cauchy's Theorem allow us to join (7) and (10) to deduce for $\operatorname{Re} \tau \leq V^{-1}(\infty)$ that

$$
\begin{aligned}
F(\tau)-\bar{v}(V)(\gamma \tau+\delta)^{k-2} e^{-2 \pi i k} F(V \tau) & \frac{1}{\Gamma(k-1)}\left\{\int_{V^{-1}(\infty)}^{i \infty}\left[f(z)-f_{\infty}(z)-\frac{f_{\infty}(V z)}{v(V)(\gamma z+\delta)^{k}}\right](z-\tau)^{k-2} d z\right. \\
& +\int_{V^{-1}(\infty)}^{-i \infty} f_{\infty}(z)(z-\tau)^{k-2} d z+\int_{\tau}^{i \infty} \frac{f_{\infty}(V z)}{v(V)(\gamma z+\delta)^{k}}(z-\tau)^{k-2} d z \\
& \left.+e^{-2 \pi i k} \int_{V^{-1}(\infty)}^{\tau} \frac{f_{\infty}(V z)}{v(V)(\gamma z+\delta)^{k}}(z-\tau)^{k-2} e^{2 \pi i N k} d z\right\}
\end{aligned}
$$

where the paths of integration are vertical lines except for the last one which is still a major arc (or union of two rays). But deformation (if needed) of this contour coupled with trivial estimates reveals that the ultimate integral (without the factor preceding it) equals

$$
\int_{V^{-1}(\infty)}^{-i \infty} \frac{f_{\infty}(V z)}{v(V)(\gamma z+\delta)^{k}}(z-\tau)^{k-2} d z-\int_{\tau}^{i \infty} \frac{f_{\infty}(V z)}{v(V)(\gamma z+\delta)^{k}}(z-\tau)^{k-2} d z
$$

and secures the proof for $\operatorname{Re} \tau \leq V^{-1}(\infty)$.

Similarly, we find for $\operatorname{Re} \tau>V^{-1}(\infty)$ that

$$
\begin{aligned}
& F(\tau)-\bar{v}(V)(\gamma \tau+\delta)^{k-2} F(V \tau) \\
& =\frac{1}{\Gamma(k-1)}\left\{\int_{V^{-1}(\infty)}^{i \infty}\left[f(z)-f_{\infty}(z)-\frac{f_{\infty}(V z)}{v(V)(\gamma z+\delta)^{k}}\right](z-\tau)^{k-2} e^{2 \pi i N_{2} k} d z\right. \\
& \quad+\int_{V^{-1}(\infty)}^{-i \infty} \frac{f_{\infty}(V z)}{v(V)(\gamma z+\delta)^{k}}(z-\tau)^{k-2} d z+\int_{\tau}^{-i \infty} f_{\infty}(z)(z-\tau)^{k-2} d z \\
& \left.\quad+\int_{V^{-1}(\infty)}^{\tau} f_{\infty}(z)(z-\tau)^{k-2} e^{2 \pi i N k} d z\right\}
\end{aligned}
$$

where $N_{2}$ is defined in Theorem 1 and the last contour is a minor arc. Rewriting and deforming yields the result wanted and concludes the proof altogether.

What about the analog of Theorem 1 for forms of lower weight? Convergence difficulties require the use of fractional differentiation. Since derivatives annihilate constants, there is no need to assume that $f$ is constant-free in 
THEOREM 2. Let $f$ be a modular form, with (possibly trivial) principal part $f_{\infty}$, of real weight $k \leq 1$, and denote by $F$ its generalized Eichler integral, given by (4). Also, let $m$ be the smallest integer greater than $1-k$. Then $F$ satisfies the relation

$$
\begin{aligned}
F(\tau)-\bar{v}(V)(\gamma \tau+\delta)^{k-2} e^{-2 \pi i N_{1} k} F(V \tau) & \frac{(-1)^{m}}{\Gamma(k+m-1)}\left\{\int_{V^{-1}(\infty)}^{i \infty} \frac{d^{m}}{d z^{m}}\left[f(z)-f_{\infty}(z)-\frac{f_{\infty}(V z)}{v(V)(\gamma z+\delta)^{k}}\right]\right. \\
& \times(z-\tau)^{k+m-2} e^{2 \pi i N_{2} k} d z \\
& +e^{-2 \pi i N_{1} k} \int_{V^{-1}(\infty)}^{-i \infty} \frac{d^{m}}{d z^{m}}\left[e^{2 \pi i k} f_{\infty}(z)+\frac{f_{\infty}(V z)}{v(V)(\gamma z+\delta)^{k}}\right](z-\tau)^{k+m-2} d z \\
& +\left(1-e^{-2 \pi i N_{1} k}\right) \int_{\tau}^{i \infty} \frac{d^{m}}{d z^{m}}\left[\frac{f_{\infty}(V z)}{v(V)(\gamma z+\delta)^{k}}\right](z-\tau)^{k+m-2} d z \\
& \left.+\left(1-e^{2 \pi i\left(1-N_{1}\right) k}\right) \int_{\tau}^{-i \infty} \frac{d^{m}}{d z^{m}}\left[f_{\infty}(z)\right](z-\tau)^{k+m-2} d z\right\}
\end{aligned}
$$

for all $V=\left(\begin{array}{cc}* & * \\ \gamma & \delta\end{array}\right) \in \Gamma(1), \gamma>0$, and $\tau \in \mathcal{H}$, where $N_{1}$ and $N_{2}$ are defined as in Theorem 1. (As before, the paths of integration are vertical.)

Proof. Except for some technical hurdles, the proof here resembles the one just given. Obvious convergence problems force us to modify the integral representation which was used for $k>1$. From (4), we see that $F$, the $(1-k)$ th fractional derivative of $f$, equals $(-1)^{m}$ times the $(k+m-1)$ th fractional integral of the $m$ th ordinary derivative of $f$. This means that

$$
\begin{aligned}
F(\tau)= & \frac{(-1)^{m}}{\Gamma(k+m-1)}\left\{\int_{\tau}^{i \infty}\left[f^{(m)}(z)-f_{\infty}^{(m)}(z)\right](z-\tau)^{k+m-2} d z\right. \\
& \left.+\int_{\tau}^{-i \infty} f_{\infty}^{(m)}(z)(z-\tau)^{k+m-2} d z\right\},
\end{aligned}
$$

which is valid for $k \leq 1$. From (11) and (9) it follows that

$$
\begin{aligned}
& -\bar{v}(V)(\gamma \tau+\delta)^{k+m-2} F(V \tau) \\
= & \frac{(-1)^{m}}{\Gamma(k+m-1)}\left\{\int_{V^{-1}(\infty)}^{\tau} \frac{f^{(m)}(V u)-f_{\infty}^{(m)}(V u)}{v(V)(\gamma u+\delta)^{k+m}}(u-\tau)^{k+m-2} e^{2 \pi i N k} d u\right. \\
& \left.+\int_{V^{-1}(\infty)}^{\tau} \frac{f_{\infty}^{(m)}(V u)}{v(V)(\gamma u+\delta)^{k+m}}(u-\tau)^{k+m-2} e^{2 \pi i N k} d u\right\},
\end{aligned}
$$


where the two paths of integration are exactly as in the proof of Theorem 1 . That is, the first contour is a hyperbolic geodesic in $\mathcal{H}$, whereas the second is its complement with respect to the generalized circle. Obviously, the value of $N$ on the two contours remains as before.

In order to marry (11) and (12) we need to rewrite the right side of the latter equality. From (1) we obtain

$$
\begin{aligned}
= & \frac{(-1)^{m}}{\Gamma(k+m-1)} \sum_{j=0}^{m}\left(\begin{array}{c}
m \\
j
\end{array}\right) \frac{\Gamma(k+m)}{\Gamma(k+j)} \gamma^{m-j} \\
& \times\left\{\int_{V^{-1}(\infty)}^{\tau}(\gamma u+\delta)^{j} \frac{d^{j}}{d u^{j}}\left[f(u)-\frac{f_{\infty}(V u)}{v(V)(\gamma u+\delta)^{k}}\right](u-\tau)^{k+m-2} e^{2 \pi i N k} d u\right. \\
& \left.+\int_{V^{-1}(\infty)}^{\tau}(\gamma u+\delta)^{j} \frac{d^{j}}{d u^{j}}\left[\frac{f_{\infty}(V u)}{v(V)(\gamma u+\delta)^{k}}\right](u-\tau)^{k+m-2} e^{2 \pi i N k} d u\right\} \\
= & \frac{(-1)^{m}}{\Gamma(k+m-1)}(\gamma \tau+\delta)^{m} \\
& \times\left\{\int_{V^{-1}(\infty)}^{\tau} \frac{d^{m}}{d u^{m}}\left[f(u)-\frac{f_{\infty}(V u)}{v(V)(\gamma u+\delta)^{k}}\right](u-\tau)^{k+m-2} e^{2 \pi i N k} d u\right. \\
& \left.+\int_{V^{-1}(\infty)}^{\tau} \frac{d^{m}}{d u^{m}}\left[\frac{f_{\infty}(V u)}{v(V)(\gamma u+\delta)^{k}}\right](u-\tau)^{k+m-2} e^{2 \pi i N k} d u\right\},
\end{aligned}
$$

where we used integration by parts (the right number of times) on each of the terms in the sum indexed by $j$. Note that each of these integrals is indeed convergent. Finally, joining (13) with (11) exactly as we did in the demonstration of Theorem 1 completes the proof.

When $f$ is a cusp form we have the following much simpler result.

COROLlary 3. Let $f$ be a cusp form of any real weight $k$ and denote by $F$ its generalized Eichler integral, given by the Fourier expansion (4). If $k>1$, then $F$ satisfies the relation

$$
\begin{aligned}
F(\tau)-\bar{v}(V)(\gamma \tau+\delta)^{k-2} e^{-2 \pi i N_{1} k} F(V \tau) & \\
= & \frac{1}{\Gamma(k-1)} \int_{V^{-1}(\infty)}^{i \infty} f(z)(z-\tau)^{k-2} e^{2 \pi i N_{2} k} d z
\end{aligned}
$$


and if $k \leq 1$, then $F$ satisfies the relation

$$
\begin{aligned}
& F(\tau)-\bar{v}(V)(\gamma \tau+\delta)^{k-2} e^{-2 \pi i N_{1} k} F(V \tau) \\
&=-\frac{1}{\Gamma(k)} \int_{V^{-1}(\infty)}^{i \infty} f^{\prime}(z)(z-\tau)^{k-1} e^{2 \pi i N_{2} k} d z
\end{aligned}
$$

Both cases hold for all $V=\left(\begin{array}{cc}* & * \\ \gamma & \delta\end{array}\right) \in \Gamma(1), \gamma>0$, and $\tau \in \mathcal{H}$, where $N_{1}$ and $\mathrm{N}_{2}$ are defined as before.

Proof. Since $f$ is constant-free and $f_{\infty} \equiv 0$, the case $k>1$ follows from Theorem 1. Similarly, the case $k \leq 1$ follows from Theorem 2 and the fact that there are no nontrivial cusp forms of nonpositive weight.

We remark that the right-hand sides of the above transformation laws are reminiscent of the periods found in Niebur's work [7]. This connection will be explored in Section 4. Furthermore, we note that to make Corollary 3 valid for all $\gamma$, it suffices to simply extend the definition of $N_{1}$ as follows:

$$
N_{1}=N_{1}(\tau, V)= \begin{cases}0 & \text { if } \gamma=0 \\ N_{1}(\tau,-V)-1 & \text { if } \gamma<0\end{cases}
$$

(This follows from a remark made after the statement of Theorem 1.)

We close this section by recording the important special case of integral weight.

COROLlary 4. Let $f$ be a modular form of integer weight $k$ and assume that $f$ is constant-free if $k>1$. Denote by $F$ its generalized Eichler integral. If $k>1$, then $F$ is an Eichler integral, which satisfies the relation

$$
\begin{aligned}
F(\tau)- & \bar{v}(V)(\gamma \tau+\delta)^{k-2} F(V \tau) \\
= & \frac{1}{(k-2) !}\left\{\int_{V^{-1}(\infty)}^{i \infty}\left[f(z)-f_{\infty}(z)-\frac{f_{\infty}(V z)}{v(V)(\gamma z+\delta)^{k}}\right](z-\tau)^{k-2} d z\right. \\
& \left.+\int_{V^{-1}(\infty)}^{-i \infty}\left[f_{\infty}(z)+\frac{f_{\infty}(V z)}{v(V)(\gamma z+\delta)^{k}}\right](z-\tau)^{k-2} d z\right\}
\end{aligned}
$$

for all $V=\left(\begin{array}{cc}* & * \\ \gamma & \delta\end{array}\right) \in \Gamma(1)$ and $\tau \in \mathcal{H}$. If $k \leq 1$, then $F$ is a modular form of weight $2-k$ and $M S v$.

Proof. The case $k>1$ follows right away from Theorem 1, whereas the case $k \leq 1$ follows readily from either Bol's Identity or from redoing the proof of Theorem 2 or from observing that the relation stated in Theorem 2 
reduces to

$$
\begin{aligned}
F(\tau)-\bar{v}(V)(\gamma \tau & +\delta)^{k-2} F(V \tau) \\
& =\int_{V^{-1}(\infty)}^{i \infty} \frac{d^{2-k}}{d z^{2-k}}\left[f(z)-f_{\infty}(z)-\frac{f_{\infty}(V z)}{v(V)(\gamma z+\delta)^{k}}\right] d z \\
& +\int_{V^{-1}(\infty)}^{-i \infty} \frac{d^{2-k}}{d z^{2-k}}\left[f_{\infty}(z)+\frac{f_{\infty}(V z)}{v(V)(\gamma z+\delta)^{k}}\right] d z
\end{aligned}
$$

and calculating that this constant function vanishes identically.

Note that the right side of the modular relation for $k>1$ is a polynomial of degree at most $k-2$. Additionally, if $f_{\infty} \not \equiv 0$, then it is known that $F+A_{0}$ could itself be a modular form. The constant term $A_{0}$ is zero except perhaps when $\kappa=0$. Under this assumption, it is arbitrary for $k=2$, but for other positive even integers it possesses a canonical value which depends upon $k$ and $f_{\infty}$. We shall probe this further below.

3. Fractional integrals of Poincaré series. When $k>2$ then it is classical knowledge that Petersson's Poincaré series

$$
f_{\nu}(\tau)=f_{\nu}(\tau ; k, v)=\frac{1}{2} \sum_{\substack{c, d \in \mathbb{Z} \\(c, d)=1}} \frac{e^{2 \pi i(\nu+\kappa) M \tau}}{v(M)(c \tau+d)^{k}}, \quad \tau \in \mathcal{H}
$$

where $\nu$ is an integer and $M=\left(\begin{array}{ll}* & * \\ c & d\end{array}\right) \in \Gamma(1)$, converges absolutely and is a modular form of weight $k$ and MS $v$ on the full modular group. If $\nu+\kappa>0$, then $f_{\nu}$ is a cusp form and the modular behavior of its generalized Eichler integral is already described fully in Corollary 3. If $\nu+\kappa=0$, then $f_{\nu}$ is a (normalized) Eisenstein series of even weight (since we are on $\Gamma(1)$ ) and its Eichler integral is rather well understood. If $\nu+\kappa<0$, then we call $f_{\nu}$ a "polar Poincaré series" and observe that it is a constant-free form which permits the following refinement of Theorem 1. In order to avoid some technical intricacies, we suppose that the multiplier system is nonsingular. As aforementioned, this restriction applies to even integer weights only.

TheOREM 5. Let $f_{\nu}, \nu<0$ and $\kappa \neq 0$, be defined by (14) and denote by $F_{\nu}$ its generalized Eichler integral. Then $F_{\nu}$ satisfies the relation

$$
\begin{aligned}
F_{\nu}(\tau) & -\bar{v}(V)(\gamma \tau+\delta)^{k-2} e^{-2 \pi i N_{1} k} F_{\nu}(V \tau) \\
\quad= & \frac{1}{\Gamma(k-1)}\left\{e^{-2 \pi i N_{1} k} \int_{V^{-1}(\infty)}^{-i \infty}\left[e^{2 \pi i k} f_{\nu}^{-}(z, V)+f_{\nu}^{+}(z, V)\right](z-\tau)^{k-2} d z\right.
\end{aligned}
$$




$$
\begin{aligned}
& +\left(1-e^{-2 \pi i N_{1} k}\right) \int_{\tau}^{i \infty} f_{\nu}^{+}(z, V)(z-\tau)^{k-2} d z \\
& \left.+\left(1-e^{2 \pi i\left(1-N_{1}\right) k}\right) \int_{\tau}^{-i \infty} f_{\nu}^{-}(z, V)(z-\tau)^{k-2} d z\right\},
\end{aligned}
$$

for all $V=\left(\begin{array}{cc}* & * \\ \gamma & \delta\end{array}\right) \in \Gamma(1), \gamma>0$, and $\tau \in \mathcal{H}$, where $N_{1}=N_{1}(\tau, V)$ is defined in Theorem 1. (Note that the paths of integration are vertical and the last term equals zero for $\operatorname{Re} \tau \leq V^{-1}(\infty)$.) Here

$$
f_{\nu}^{-}(w, V)=f_{\nu}^{-}(w, V ; k, v)=e^{2 \pi i(\nu+\kappa) w}+\sum_{\substack{c, d \in \mathbb{Z}, c>0 \\(c, d)=1 \\ M^{-1}(\infty)<V^{-1}(\infty)}} \frac{e^{2 \pi i(\nu+\kappa) M w}}{v(M)(c w+d)^{k}}
$$

with $w \in \mathbb{C} \backslash\left(-\infty, V^{-1}(\infty)\right)$, and

$$
f_{\nu}^{+}(w, V)=f_{\nu}^{+}(w, V ; k, v)=\sum_{\substack{c, d \in \mathbb{Z}, c>0 \\(c, d)=1 \\ M^{-1}(\infty) \geq V^{-1}(\infty)}} \frac{e^{2 \pi i(\nu+\kappa) M w}}{v(M)(c w+d)^{k}}
$$

with $w \in \mathbb{C} \backslash\left[V^{-1}(\infty), \infty\right)$.

We remark that $f_{\nu}^{-}(w, V)$ is holomorphic in $\mathbb{C} \backslash\left(-\infty, V^{-1}(\infty)\right]$, whereas $f_{\nu}^{+}(w, V)$ is holomorphic in $\mathbb{C} \backslash \mathbb{R}$ only. This is a clear consequence of our argument convention. We could remove this asymmetry by altering the definition of $f_{\nu}^{+}$. (Just replace the condition $c>0$ with $c<0$ to get a function which is holomorphic in $\mathbb{C} \backslash\left[V^{-1}(\infty), \infty\right)$. The two functions coincide in $\mathcal{H}$, but differ by a constant factor in $\overline{\mathcal{H}}$.) Ultimately, however, the results would be equivalent.

Proof of Theorem 5. Once again, we shall rely on Theorem 1. Observe that for $w \in \mathcal{H}$,

$$
f_{\nu}(w)=e^{2 \pi i(\nu+\kappa) w}+\sum_{\substack{c, d \in \mathbb{Z}, c>0 \\(c, d)=1}} \frac{e^{2 \pi i(\nu+\kappa) M w}}{v(M)(c w+d)^{k}}=f_{\nu}^{-}(w, V)+f_{\nu}^{+}(w, V)
$$

and so

$$
\begin{gathered}
\int_{V^{-1}(\infty)}^{i \infty}\left[f_{\nu}(z)-e^{2 \pi i(\nu+\kappa) z}-\frac{e^{2 \pi i(\nu+\kappa) V z}}{v(V)(\gamma z+\delta)^{k}}\right](z-\tau)^{k-2} e^{2 \pi i N_{2} k} d z \\
=\int_{V^{-1}(\infty)}^{i \infty}\left[f_{\nu}^{-}(z, V)-e^{2 \pi i(\nu+\kappa) z}\right](z-\tau)^{k-2} e^{2 \pi i N_{2} k} d z
\end{gathered}
$$




$$
+\int_{V^{-1}(\infty)}^{i \infty}\left[f_{\nu}^{+}(z, V)-\frac{e^{2 \pi i(\nu+\kappa) V z}}{v(V)(\gamma z+\delta)^{k}}\right](z-\tau)^{k-2} e^{2 \pi i N_{2} k} d z
$$

( $N_{2}$ is defined in Theorem 1.) Denote the last two integrals by $I_{1}$ and $I_{2}$, respectively. We can rewrite them by deforming their contours. For $\operatorname{Re} \tau \leq$ $V^{-1}(\infty)$, which implies that $N_{2}=0$, we rotate the first contour clockwise (about the fixed point $z=V^{-1}(\infty)$ ) to get

$$
I_{1}=\int_{V^{-1}(\infty)}^{-i \infty}\left[f_{\nu}^{-}(z, V)-e^{2 \pi i(\nu+\kappa) z}\right](z-\tau)^{k-2} d z
$$

whereas we bend the second path counterclockwise to obtain

$$
\begin{aligned}
I_{2}= & e^{-2 \pi i k} \int_{V^{-1}(\infty)}^{-i \infty}\left[f_{\nu}^{+}(z, V)-\frac{e^{2 \pi i(\nu+\kappa) V z}}{v(V)(\gamma z+\delta)^{k}}\right](z-\tau)^{k-2} d z \\
& +\left(1-e^{-2 \pi i k}\right) \int_{\tau}^{i \infty}\left[f_{\nu}^{+}(z, V)-\frac{e^{2 \pi i(\nu+\kappa) V z}}{v(V)(\gamma z+\delta)^{k}}\right](z-\tau)^{k-2} d z .
\end{aligned}
$$

Note that the first term in $I_{2}$ took into account the jump discontinuity associated with crossing the real axis (itself the union of the infinitude of overlapping branch cuts emanating from points to the right of $z=V^{-1}(\infty)$ ) and the second term in $I_{2}$ arose from dealing with the branch point at $z=\tau$. Similarly, for $\operatorname{Re} \tau>V^{-1}(\infty)$ we push the first path clockwise to get

$$
\begin{aligned}
I_{1}= & e^{2 \pi i k} \int_{V^{-1}(\infty)}^{-i \infty}\left[f_{\nu}^{-}(z, V)-e^{2 \pi i(\nu+\kappa) z}\right](z-\tau)^{k-2} d z \\
& +\left(1-e^{2 \pi i k}\right) \int_{\tau}^{-i \infty}\left[f_{\nu}^{-}(z, V)-e^{2 \pi i(\nu+\kappa) z}\right](z-\tau)^{k-2} d z
\end{aligned}
$$

and the second contour counterclockwise to produce

$$
I_{2}=\int_{V^{-1}(\infty)}^{-i \infty}\left[f_{\nu}^{+}(z, V)-\frac{e^{2 \pi i(\nu+\kappa) V z}}{v(V)(\gamma z+\delta)^{k}}\right](z-\tau)^{k-2} d z,
$$

where in both cases we used the definition of $N_{2}$. Observe that as $|z| \rightarrow \infty$ in the appropriate regions pertaining to our deformations, all of the above integrands approach zero in a manner that permits us to rotate contours with impunity. (Here the condition that $\kappa \neq 0$ is crucial!) The rest of the proof follows without difficulty from Theorem 1 in combination with our new expressions for $I_{1}$ and $I_{2}$.

As a welcome byproduct of our work, we rediscover the following pretty result of Knopp [3] concerning the interrelationships of period polynomials connected with Eichler integrals of Poincaré series. 
Corollary 6. Let $f_{\nu}, \kappa \neq 0$, be a Poincaré series of integer weight $k>2$ and denote its Eichler integral by $F_{\nu}$. Furthermore, let

$$
p_{V}(\tau ; \nu, k, v)=F_{\nu}(\tau)-\bar{v}(V)(\gamma \tau+\delta)^{k-2} F_{\nu}(V \tau),
$$

where $V=\left(\begin{array}{ll}* & * \\ \gamma & \delta\end{array}\right) \in \Gamma(1)$, be the associated period polynomials. Then for all $V$,

$$
p_{V}(\tau ; \nu, k, v)=\left[p_{V}\left(\bar{\tau} ; \nu^{\prime}, k, \bar{v}\right)\right]^{-}, \quad \tau \in \mathbb{C},
$$

where $\nu^{\prime}=-\nu-1$ and $[\cdot]^{-}$denotes complex conjugation.

Proof. If $\nu+\kappa>0$, then clearly

$$
p_{V}(\tau ; \nu, k, v)=\frac{1}{(k-2) !} \int_{V^{-1}(\infty)}^{i \infty} f_{\nu}(z)(z-\tau)^{k-2} d z,
$$

and if $\nu+\kappa<0$, then Theorem 5 tells us that

$$
\begin{aligned}
p_{V}(\tau ; \nu, k, v) & \\
& =\frac{1}{(k-2) !} \int_{V^{-1}(\infty)}^{-i \infty}\left[f_{\nu}^{-}(z, V)+f_{\nu}^{+}(z, V)\right](z-\tau)^{k-2} d z \\
& =\frac{1}{(k-2) !}\left\{\int_{V^{-1}(\infty)}^{i \infty}\left[\overline{f_{\nu}^{-}(\bar{u}, V)}+\overline{f_{\nu}^{+}(\bar{u}, V)}\right](u-\bar{\tau})^{k-2} d u\right\}^{-} \\
& =\frac{1}{(k-2) !}\left[\int_{V^{-1}(\infty)}^{i \infty} f_{\nu^{\prime}}(z ; k, \bar{v})(z-\bar{\tau})^{k-2} d z\right]^{-}
\end{aligned}
$$

where we used the key identity

$$
\overline{f_{\nu}^{-}(\bar{w}, V)}+\overline{f_{\nu}^{+}(\bar{w}, V)}=f_{\nu^{\prime}}^{-}(w, V ; k, \bar{v})+f_{\nu^{\prime}}^{+}(w, V ; k, \bar{v})=f_{\nu^{\prime}}(w ; k, \bar{v}),
$$

which is valid for $w \in \mathcal{H}$. The proof now follows from comparison of (15) and (16).

Our proof of the above result (by way of integral representations and contour bending) appears to be new. A more delicate version of our analysis can be performed to handle the case of Knopp's Theorem when the parameter $\kappa$ vanishes. In this case it follows that for all $V \in \Gamma(1)$ and $\nu<0$,

$$
p_{V}(\tau ; \nu, k, 1)=\left[p_{V}\left(\bar{\tau} ; \nu^{\prime}, k, 1\right)\right]^{-}-A_{0}(\nu, k)\left[1-(\gamma \tau+\delta)^{k-2}\right], \quad \tau \in \mathbb{C},
$$

where $\nu^{\prime}=-\nu$ and $A_{0}(\nu, k)$ is the canonical nonzero constant which emerges. This relation tells us that for $\nu<0$ and $\kappa=0$, it is appropriate to define the Eichler integral of $f_{\nu}$ by $F_{\nu}+A_{0}(\nu, k)$. This allows for the possibility that the integral is actually a form. 
4. Niebur modular integrals. A Niebur modular integral on $\Gamma(1)$ of weight $k$ and $M S v$ is a function $H$ which is holomorphic in $\mathcal{H}$, meromorphic at $\infty$, and for which there exists a cusp form $G$ of weight $2-k$ and MS $\bar{v}$ such that

$$
H(\tau)-\bar{v}(V)(\gamma \tau+\delta)^{-k} H(V \tau)=\left[\int_{V^{-1}(\infty)}^{i \infty} G(z)(z-\bar{\tau})^{-k} d z\right]^{-}
$$

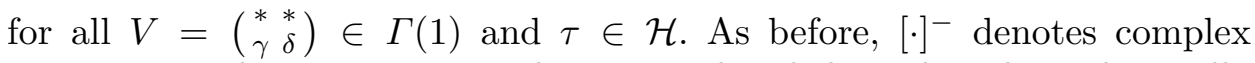
conjugation, the integration path is vertical and the right side is identically zero if $V$ is a translation. Clearly, this definition accomodates all modular forms (of any weight) as well as certain Eichler integrals of integer weight. Specifically, it includes the Eichler integrals of forms which are finite linear combinations of polar Poincaré series. (To see this, examine Niebur's original work [7].) For noninteger weights, it is transparent that a generalized Eichler integral is not a Niebur modular integral. This stems from the fact that for such weights, $v$ and $\bar{v}$ cannot simultaneously be multiplier systems with respect to $\Gamma(1)$.

We now provide a simple link between polar Poincaré series of real weight and Niebur modular integrals of dual weight. Let $f_{\nu}, \nu<0$ and $\kappa \neq 0$, be defined by (14), so that

$$
f_{\nu}(\tau)=e^{2 \pi i(\nu+\kappa) \tau}+\sum_{n+\kappa>0} a_{n}(\nu, k, v) e^{2 \pi i(n+\kappa) \tau}, \quad \tau \in \mathcal{H},
$$

is its Fourier expansion. We define the conjugate Eichler integral of $f_{\nu}$ by

$$
\begin{aligned}
F_{\nu}^{*}(\tau)= & \frac{e^{2 \pi i\left(\nu+\kappa^{\prime}\right) \tau}}{\left[-2 \pi i\left(\nu+\kappa^{\prime}\right)\right]^{k-1}} \\
& +\sum_{n+\kappa^{\prime}>0} \frac{a_{n}(\nu, k, \bar{v})}{\left[-2 \pi i\left(n+\kappa^{\prime}\right)\right]^{k-1}} e^{2 \pi i\left(n+\kappa^{\prime}\right) \tau},
\end{aligned}
$$

where $\tau \in \mathcal{H}$ and $\kappa^{\prime}=1-\kappa$. (The case $\kappa=0$ was already discussed at the end of the previous section.) Observe that when $k$ is an integer, then of course $F_{\nu}^{*}$ is itself an Eichler integral. In fact, if its "supplementary Fourier series" (as defined by Knopp [3]) vanishes identically, then $F_{\nu}^{*}$ is a modular form. For general $k$ note that the conjugate Eichler integral is merely the generalized Eichler integral with the multiplier system conjugated. That is, $v$ gets replaced by $\bar{v}$ and so $\kappa$ gets replaced by $\kappa^{\prime}$.

Furthermore, the two operations of fractional integration ( $k-1$ times) and conjugation of multiplier system are commutative. But we caution that the validity of this statement evaporates if we work with the original Poincaré series for $f_{\nu}$ rather than its Fourier expansion. To see this note 
that

$$
\begin{aligned}
& \frac{1}{2} \sum_{\substack{c, d \in \mathbb{Z} \\
(c, d)=1}} \frac{e^{2 \pi i\left(\nu+\kappa^{\prime}\right) M \tau}}{\bar{v}(M)(c \tau+d)^{k}} \\
& =\frac{\left(1+e^{2 \pi i k}\right)}{2}\left\{e^{2 \pi i\left(\nu+\kappa^{\prime}\right) \tau}+\sum_{\substack{c, d \in \mathbb{Z}, c>0 \\
(c, d)=1}} \frac{e^{2 \pi i\left(\nu+\kappa^{\prime}\right) M \tau}}{\bar{v}(M)(c \tau+d)^{k}}\right\},
\end{aligned}
$$

where we used (6) and the fact that $\bar{v}(-I)(-1)^{k}=e^{-2 \pi i k}$, which follows from the condition $v(-I)(-1)^{k}=1$. Since the above series vanishes identically for half-integer weights, it is rather evident that in general commutativity does not hold if the operators act on the original Poincaré series. For integer weights it remains true, since then (and only then) both $v$ and $\bar{v}$ are multiplier systems. The situation can be rectified easily, of course, by starting with the series

$$
e^{2 \pi i(\nu+\kappa) \tau}+\sum_{\substack{c, d \in \mathbb{Z}, c>0 \\(c, d)=1}} \frac{e^{2 \pi i(\nu+\kappa) M \tau}}{v(M)(c \tau+d)^{k}},
$$

which is equivalent to our previous formulation which commenced with the Fourier expansion for $f_{\nu}$.

We now provide the main upshot of this section.

Theorem 7. Let $f_{\nu}, \nu<0$ and $\kappa \neq 0$, be a Poincaré series of weight $k>2$ and denote by $F_{\nu}^{*}$ its conjugate Eichler integral, given by the Fourier expansion (18). Then $F_{\nu}^{*}$ is a Niebur modular integral of weight $2-k$ and $M S \bar{v}$.

Proof. The proof follows quickly from known explicit formulas for the Fourier coefficients of the relevant functions. Specifically, Petersson [8] showed that the Fourier expansion of the polar $f_{\nu}$ is given by $(17)$, where

$$
\begin{aligned}
a_{n}(\nu, k, v)= & 2 \pi i^{-k}\left(\frac{n+\kappa}{|\nu+\kappa|}\right)^{(k-1) / 2} \\
& \times \sum_{c=1}^{\infty} \frac{A(c ; \nu, n, k, v)}{c} I_{k-1}\left(\frac{4 \pi}{c} \sqrt{|\nu+\kappa|(n+\kappa)}\right) .
\end{aligned}
$$

Here $I_{k-1}$ is the modified Bessel function of the first kind and

$$
A(c ; \nu, n, k, v)=\sum_{\substack{-d=0 \\(c, d)=1}}^{c-1} \bar{v}(M) e^{(2 \pi i / c)[(\nu+\kappa) a+(n+\kappa) d]},
$$

with $M=\left(\begin{array}{ll}a & * \\ c & d\end{array}\right) \in \Gamma(1)$, is the generalized Kloosterman sum. From this and 
(18) we obtain the (normalized) conjugate Eichler integral

where

$$
\left[-2 \pi i\left(\nu+\kappa^{\prime}\right)\right]^{k-1} F_{\nu}^{*}(\tau)=e^{2 \pi i\left(\nu+\kappa^{\prime}\right) \tau}+\sum_{n+\kappa^{\prime}>0} a_{n}(\nu, 2-k, \bar{v}) e^{2 \pi i\left(n+\kappa^{\prime}\right) \tau},
$$

$$
\begin{aligned}
a_{n}(\nu, 2-k, \bar{v})= & 2 \pi i^{k-2}\left(\frac{\left|\nu+\kappa^{\prime}\right|}{n+\kappa^{\prime}}\right)^{(k-1) / 2} \\
& \times \sum_{c=1}^{\infty} \frac{A(c ; \nu, n, 2-k, \bar{v})}{c} I_{k-1}\left(\frac{4 \pi}{c} \sqrt{\left|\nu+\kappa^{\prime}\right|\left(n+\kappa^{\prime}\right)}\right) .
\end{aligned}
$$

But Niebur [7] proved that this expansion, which sometimes belongs to a modular form (see [13]), always represents a modular integral of the type he discovered.

With some modifications, Theorem 7 can be extended to smaller real weights. In order to accomplish this, we need to consider the nonanalytic Poincaré series

$$
f_{\nu}(\tau \mid s)=f_{\nu}(\tau \mid s ; k, v)=\frac{1}{2} \sum_{\substack{c, d \in \mathbb{Z} \\(c, d)=1}} \frac{e^{2 \pi i(\nu+\kappa) M \tau}}{v(M)(c \tau+d)^{k}|c \tau+d|^{s}}, \quad \tau \in \mathcal{H},
$$

where $\operatorname{Re} s>2-k, k$ is real and all other notation remains as before. It is known from the work of Selberg [14] that $f_{\nu}(\tau \mid s)$ has an analytic continuation to a function which is meromorphic in the whole $s$-plane. Moreover, it was established in [10] that for $\nu<0$ and $1<k<2$ the special value $f_{\nu}(\tau):=f_{\nu}(\tau \mid 0)$, which transforms like a modular form of weight $k$ and MS $v$, decomposes naturally into a sum of an analytic piece $g_{\nu}(\tau)$ and a remaining piece $r_{\nu}(\tau)$ which is either nonanalytic or vanishes identically. It turns out that $r_{\nu}$ transforms like a Niebur modular integral of weight $k$ and MS $v$. It is an "auxiliary integral", a type of function which we examine briefly in the next section. This implies that $g_{\nu}$ is a Niebur modular integral of weight $k$ and MS $v$ on the full modular group. The cusp form associated with $g_{\nu}$ is the negative of the cusp form connected with $r_{\nu}$. Furthermore, if $r_{\nu}$ vanishes identically, then of course $g_{\nu} \equiv f_{\nu}$ is a modular form. (For details on all of this, see [10].) Next, let the Fourier expansion of $g_{\nu}, \nu<0$ and $1<k<2$, be given by

$$
g_{\nu}(\tau)=e^{2 \pi i(\nu+\kappa) \tau}+\sum_{n+\kappa>0} b_{n}(\nu, k, v) e^{2 \pi i(n+\kappa) \tau}, \quad \tau \in \mathcal{H}
$$

In complete analogy to how we handled polar Poincaré series, define the conjugate Eichler integral of $g_{\nu}$ by

$$
G_{\nu}^{*}(\tau)=\frac{e^{2 \pi i\left(\nu+\kappa^{\prime}\right) \tau}}{\left[-2 \pi i\left(\nu+\kappa^{\prime}\right)\right]^{k-1}}+\sum_{n+\kappa^{\prime}>0} \frac{b_{n}(\nu, k, \bar{v})}{\left[-2 \pi i\left(n+\kappa^{\prime}\right)\right]^{k-1}} e^{2 \pi i\left(n+\kappa^{\prime}\right) \tau},
$$

where $\tau \in \mathcal{H}$ and $\kappa^{\prime}$ is defined as before. We have explained enough to state 
THEOREM 8. Let $g_{\nu}, \nu<0$, be the Niebur modular integral of weight $k$, $1<k<2$, which is described above, and denote by $G_{\nu}^{*}$ its conjugate Eichler integral, given by the Fourier expansion (19). Then $G_{\nu}^{*}$ is a Niebur modular integral of weight $2-k$ and $M S \bar{v}$.

Proof. As before, the proof follows right away from explicit formulas for the Fourier coefficients of Niebur modular integrals of weight $k$ and $2-k$. The formula for $g_{\nu}$ is derived in [10]. From this we get the required expansion for $G_{\nu}^{*}$, which in turn jives exactly with the formula presented in [11] for a Niebur modular integral of weight $2-k$.

We remark that it is not possible for both $g_{\nu}$ and $G_{\nu}^{*}$ to be Niebur modular integrals which are not modular forms. Otherwise, the product of the two associated cusp forms would itself be a nontrivial cusp form of weight two and MS identically one. But on the full modular group this is not tenable! Observe that the other three possibilities (form \& form, form $\&$ nonform and nonform \& form) are certainly possible and in fact do occur infinitely often for every $k \& 2-k$ pairing, where $1<k<2$. For weight $k=3 / 2$ this statement can be formulated rather precisely as to which multiplier systems and values of $\nu$ provide which possibility. The interested reader should consult $[10$, Sect. 11$]$.

5. Auxiliary integrals. We mention lastly yet another type of integral connected with modular forms. Let $f$ be a cusp form of weight $k>0$ and MS $v$ on $\Gamma(1)$ and define its auxiliary integral by

$$
\mathcal{F}(\tau)=\left[\int_{\tau}^{i \infty} f(z)(z-\bar{\tau})^{k-2} d z\right]^{-}, \quad \tau \in \mathcal{H} .
$$

The function $e^{2 \pi i \kappa} \mathcal{F}$ is periodic and it is not difficult to show that

$$
\mathcal{F}(\tau)=\sum_{n+\kappa>0} \frac{\bar{a}_{n}}{[2 \pi i(n+\kappa)]^{k-1}} \int_{4 \pi(n+\kappa) y}^{\infty} u^{k-2} e^{-u} d u e^{-2 \pi i(n+\kappa) \tau},
$$

where $a_{n}$ is the $n$th Fourier coefficient of $f$ and $y=\operatorname{Im} \tau$. The integral in this Fourier series is an incomplete gamma function. Observe that, for nontrivial $f, \mathcal{F}$ is a nonanalytic function. Since there are no branching difficulties to contend with, it is straightforward to find its behavior under modular transformations. Specifically,

$$
\mathcal{F}(\tau)-v(V)(\gamma \tau+\delta)^{k-2} \mathcal{F}(V \tau)=\left[\int_{V^{-1}(\infty)}^{i \infty} f(z)(z-\bar{\tau})^{k-2} d z\right]^{-}
$$

for all $V=\left(\begin{array}{ll}* & * \\ \gamma & \delta\end{array}\right) \in \Gamma(1)$ and $\tau \in \mathcal{H}$. This means that $\mathcal{F}$ transforms like a Niebur modular integral of weight $2-k$ and MS $\bar{v}$. Furthermore, it can be 
shown that if $\nu<0 \& \kappa \neq 0$, then $F_{\nu}^{*}$, the conjugate Eichler integral of the Poincaré series $f_{\nu}$, has the same period (modulo a possible constant factor) as the auxiliary integral of the Poincaré series $f_{-\nu-1}$. As we explained somewhat in the previous section, both Niebur modular integrals and auxiliary integrals arise naturally in the study of nonanalytic Poincaré series.

6. Concluding remarks. The aim of our investigation is to understand the basic properties of fractional integrals of automorphic forms. On the full modular group we have discussed the transformation laws of the pertinent integrals. Although these relations are somewhat involved, the Fourier expansions of generalized Eichler integrals are not dramatically altered from the original series. This provides us with hope that there may be some interesting arithmetic consequences connected with these integrals. We have also seen how they are tied to Niebur modular integrals, which do possess a reasonable period structure. Our work is easily translated to other groups, although there sometimes more needs to be said (for example, integrals of arbitrary powers of the theta function come into play). We finish with a short list of items (some obvious) that we wish to address in a future article.

(1) Study generalized Eichler integrals on groups which support Eisenstein series of noninteger weight. In particular, this entails modifying our transformation law to include the generalized Eichler integrals of forms with constant terms.

(2) Determine which algebraic combinations of generalized Eichler integrals are automorphic forms. More generally, find all combinations of fractional integrals (of any order) of forms which are themselves forms. This would provide a vast generalization of the Rankin-Cohen bracket. In special cases, this should have some connection with known results about the Fourier coefficients of Jacobi forms.

(3) Provide an alternative proof of Theorems $7 \& 8$ (which show that conjugate Eichler integrals are Niebur modular integrals) without resorting to known Fourier expansions, but rather through manipulation of Poincaré series. This would provide a natural and simple derivation of (nonanalytic) pseudo-Poincaré series, which were inspired by Niebur [7] and are studied in $[11,12]$.

(4) Define and examine the conjugate Eichler integral of a cuspidal Poincaré series, and (when appropriate) of an Eisenstein series. This would lead to a generalized Niebur modular integral and a new pseudo-Poincaré series connected with it.

Acknowledgements. The author thanks Marvin Knopp and Don Zagier for their positive influence. He also thanks the referee for making insightful comments and suggestions. 


\section{References}

[1] G. Bol, Invarianten linearer Differentialgleichungen, Abh. Math. Sem. Univ. Hamburg 16 (1949), 1-28.

[2] M. Eichler, Eine Verallgemeinerung der Abelschen Integrale, Math. Z. 67 (1957), 267-298.

[3] M. Knopp, Construction of automorphic forms on H-groups and supplementary Fourier series, Trans. Amer. Math. Soc. 103 (1962), 168-188; Correction, ibid. 106 (1963), 341-345.

[4] W. Kohnen and D. Zagier, Modular forms with rational periods, in: Modular Forms (Durham, 1983), Ellis Horwood Ser. Math. Appl. Statist. Oper. Res., Horwood, Chichester, 1984, 197-249.

[5] H. Maass, Die Differentialgleichungen in der Theorie der elliptischen Modulfunktionen, Math. Ann. 125 (1953), 235-263.

[6] K. S. Miller and B. Ross, An Introduction to the Fractional Calculus and Fractional Differential Equations, Wiley, New York, 1993.

[7] D. Niebur, Construction of automorphic forms and integrals, Trans. Amer. Math. Soc. 191 (1974), 373-385.

[8] H. Petersson, Theorie der automorphen Formen beliebiger reeller Dimension und ihre Darstellung durch eine neue Art Poincaréscher Reihen, Math. Ann. 103 (1930), 369-436.

[9] W. Pribitkin, The Fourier coefficients of modular forms and modular integrals having small positive weight, doctoral dissertation, Temple Univ., Philadelphia, 1995.

[10] - The Fourier coefficients of modular forms and Niebur modular integrals having small positive weight, I, Acta Arith. 91 (1999), 291-309.

[11] —, The Fourier coefficients of modular forms and Niebur modular integrals having small positive weight, II, ibid. 93 (2000), 343-358.

[12] - Revisiting Rademacher's formula for the partition function $p(n)$, Ramanujan J. 4 (2000), 455-467.

[13] H. Rademacher and H. S. Zuckerman, On the Fourier coefficients of certain modular forms of positive dimension, Ann. of Math. 39 (1938), 433-462.

[14] A. Selberg, On the estimation of Fourier coefficients of modular forms, in: Theory of Numbers, Proc. Sympos. Pure Math. 8, Amer. Math. Soc., Providence, RI, 1965, $1-15$.

[15] G. Shimura, Sur les intégrales attachées aux formes automorphes, J. Math. Soc. Japan 11 (1959), 291-311.

[16] H. Weyl, Bemerkungen zum Begriff des Differentialquotienten gebrochener Ordnung, Vierteljahresschr. Naturforsch. Ges. Zürich 62 (1917), 296-302.

[17] D. Zagier, Vassiliev invariants and a strange identity related to the Dedekind etafunction, Topology 40 (2001), 945-960.

[18] - Period functions for modular forms of half-integral weight, in preparation.

Department of Mathematics

College of Staten Island, CUNY

2800 Victory Boulevard

Staten Island, NY 10314, U.S.A.

E-mail: pribitkin@mail.csi.cuny.edu

w_pribitkin@msn.com

Received on 9.2.2004

and in revised form on 29.6.2004 